\title{
Truncations in the amino terminus reveal a region key to supporting amphetamine-induced efflux by the human serotonin transporter
}

\author{
Sonja Sucic, Carina Kern, Dragana Vidovic, Subhodeep Sarker, Oliver Kudlacek, Harald H Sitte, Michael Freissmuth* \\ From 17th Scientific Symposium of the Austrian Pharmacological Society (APHAR). Joint meeting with the \\ Hungarian Society of Experimental and Clinical Pharmacology (MFT) \\ Innsbruck, Austria. 29-30 September 2011
}

\section{Background}

The serotonin transporter (SERT) terminates neurotransmission via reuptake of serotonin from the synaptic cleft. Upon stimulation with amphetamines, SERT switches into an outward transport mode to rapidly release serotonin. We have previously shown that truncation of the first 64 residues of SERT amino terminus leads to loss of amphetamine-induced efflux [1]. This was comparable to the effects of a single point mutation of a juxtamembrane threonine residue at position 81 [1].

\section{Methods}

Truncation mutants of SERT amino terminus were generated by removing $22(\Delta 22$-SERT), $32(\Delta 32$-SERT) or $42(\triangle 42$-SERT) amino terminal residues. In addition, alanine scanning mutagenesis was performed along a segment of amino acid residues 32-42. All mutants were pharmacologically characterised in uptake, binding and efflux studies.

\section{Results}

Cellular localisation of the mutants examined by confocal microscopy, revealed no differences compared to the wild-type SERT. Functional analysis showed only modest changes in their substrate uptake properties (no significant changes in the $K_{m}$ values and a moderate decrease in the $V_{\max }$ value of $\triangle 42$-SERT). Similarly, there were no marked alterations in the $K_{D}$ and $B_{\max }$ values of imipramine or in the $K_{i}$ values of $p$-chloroamphetamine and ibogaine, determined in radiolabelled imipramine binding assays. However, while amphetamine-induced efflux was unimpaired for $\triangle 22$-SERT and to a slight extent decreased for $\triangle 32-$ SERT, it was completely abolished for $\triangle 42$-SERT.

\section{Conclusions}

Our results shed new light on the functional role of the amino terminus and point to the segment encompassing residues $32-42$ as a region of key importance to supporting amphetamine-induced efflux by SERT.

\section{Acknowledgements}

This work was supported by SFB35.

Published: 5 September 2011

\section{Reference}

1. Sucic $S$, Dallinger $S$, Zdrazil B, Weissensteiner $R$, Jørgensen TN, Holy M, Kudlacek O, Seidel S, Cha JH, Gether U, Newman AH, Ecker GF, Freissmuth $\mathrm{M}$, Sitte $\mathrm{HH}$ : The $\mathrm{N}$ terminus of monoamine transporters is a lever required for the action of amphetamines. J Biol Chem 2010, 285:10924-10938.

\section{doi:10.1186/1471-2210-11-S2-A20}

Cite this article as: Sucic et al:: Truncations in the amino terminus reveal a region key to supporting amphetamine-induced efflux by the human serotonin transporter. BMC Pharmacology 2011 11(Suppl 2):A20.

\footnotetext{
* Correspondence: michael.freissmuth@meduniwien.ac.at Institute of Pharmacology, Center of Physiology and Pharmacology, Medical University of Vienna, 1090 Vienna, Austria
} 